\title{
Entrelacs
}

ENTRELACS Cinéma et audiovisuel

13 | 2017

Espace, perspective et fragmentation

\section{Perspective et constructions}

Jean-Luc ANTONUCCI

\section{OpenEdition}

Journals

Édition électronique

URL : http://journals.openedition.org/entrelacs/2023

DOI : 10.4000/entrelacs.2023

ISSN : 2261-5482

Éditeur

Éditions Téraèdre

\section{Référence électronique}

Jean-Luc ANTONUCCI, «Perspective et constructions », Entrelacs [En ligne], 13 | 2017, mis en ligne le

18 mai 2017, consulté le 19 avril 2019. URL : http://journals.openedition.org/entrelacs/2023 ; DOI :

10.4000/entrelacs.2023

Ce document a été généré automatiquement le 19 avril 2019

Tous droits réservés 


\title{
Perspective et constructions
}

\author{
Jean-Luc ANTONUCCI
}

1 Les images réalisées avec un appareil photographique ou une caméra pour être tirées sur papier ou projetées sur un écran sont soumises aux règles de la construction perspective des images planes. Très tôt, les réalisateurs et décorateurs de cinéma ont donc dû tenir compte des caractéristiques optiques de ces outils et des propriétés de la perspective à laquelle ils étaient confrontés. C'est pourquoi, dès le début du siècle dernier, l'industrie naissante du cinéma a fait appel au savoir-faire des peintres-décorateurs de théâtre qui avaient une parfaite maîtrise de la mise en perspective et des techniques de représentation de l'espace sur des panneaux décor. En France, ce fut notamment le cas du décorateur Hugues Laurent qui travailla pour les studios français Pathé ${ }^{1}$.

2 À un moment où l'imagerie numérique prenait de plus en plus de place dans la production cinématographique, Daniel Guéret O'Nillon, décorateur notamment du film de Jean-Pierre Melville Le Doulos, a voulu mettre en évidence un aspect particulier de ce savoir-faire des décorateurs de cinéma. Il a conçu et fait réaliser une exposition qui avait pour but de montrer que les décorateurs de cinéma étaient également des professionnels des effets spéciaux. Ce manifeste était principalement constitué d'une série de maquettes qui présentaient différents procédés de trucages associant des dispositifs optiques au travail du décor. Elle était complétée par une suite de panneaux présentant les principales propriétés de la perspective et montrant que ces dispositifs, comme le travail du décor de cinéma en général, reposaient sur la connaissance et la maitrise de la perspective ${ }^{2}$. Cette exposition présentée pour la première fois à Joinville-le-Pont, en février 1984, mettait donc en scène des maquettes d'effets spéciaux dans lesquelles le travail du décor intervenait. Devant chacune d'elles une caméra était disposée afin que les spectateurs puissent voir sur un moniteur l'image qui était obtenue.
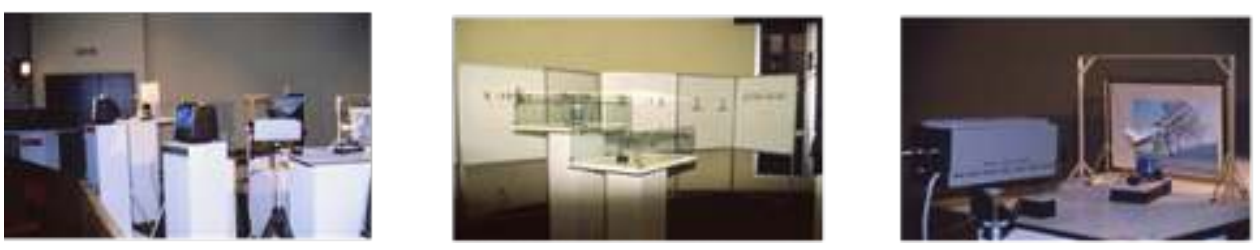

Photos Jean-Luc Antonucci 
- Une "façade couchée " (ou inclinée), permettant de filmer des acteurs grimpant une paroi verticale alors qu'ils rampent sur le décor. Le vide et le fond supposés être sous eux étant réalisés sur un panneau vertical posé à l'arrière du dispositif, face à la caméra, ou réalisé en incrustation avec un fond vert. - Un « tambour » cylindrique, en l'occurrence vertical, mais qui peut aussi être horizontal ou une combinaison de deux cylindres sur lesquels est tendue une toile, permettant de faire défiler une découverte peinte ou photographique à l'arrière d'un élément de décor fixe (ici un habitacle d'hélicoptère) afin de simuler un déplacement à l'arrière de ce décor.

6 - Une « projection frontale » (sur écran scotch light), qui donne la possibilité de combiner un élément de décor à l'échelle 1 avec des images projetées frontalement à l'aide d'un projecteur synchronisé avec la caméra et d'un miroir semi-aluminé.

7 - Une « transparence » à travers un écran placé derrière le décor, le projecteur étant placé à l'arrière de l'écran permettant également de combiner un décor avec des images projetées.

8 - Une " chambre qui tourne " construite dans un ensemble décor mobile que l'on peut faire pivoter, la caméra étant solidaire du décor. Lorsque le décor tourne, les acteurs peuvent marcher, ou danser, sur les cloisons ou le plafond au gré de la rotation du dispositif décor.

9 - Une " maquette suspendue ", intercalée entre l'action filmée et la caméra, qui va modifier ou compléter l'image et le décor. Un procédé analogue peut être réalisé avec un panneau peint découpé ou une glace peinte.

10 - Une " piscine de studio » associant maquettes, éléments de décor et cyclo peint ou photographique dans laquelle le réalisateur pourra maîtriser tout à la fois le vent, l'eau, la lumière, la position de la caméra et le jeu des acteurs.

11 Toutes ces maquettes mettaient en évidence que les images produites avec les outils de représentation audiovisuelle (photo, vidéo et cinéma) respectaient trois principes fondamentaux : la fragmentation visuelle et spatiale, la combinaison et l'absorption des intervalles entre plusieurs composants participant à la construction de l'image, la construction perspective de l'image assurant la mise en cohérence de l'image produite. En effet, pour que les images ainsi réalisées puissent être perçues correctement par les spectateurs, il est indispensable de maîtriser l'ensemble des techniques de représentation qui sont mises en œuvre afin que tous les éléments intervenant dans la composition de l'image coïncident parfaitement.

12 C'est en s'appuyant sur les propriétés de la construction perspective des images que les différentes catégories de techniciens intervenant sur un tournage vont assurer cette nécessaire cohérence.

\section{Mise en perspective}

13 Les outils de capture d'images fixes et animées sont soumis aux principes de la représentation perspective elle-même régie par l'optique géométrique. Si l'on fait abstraction des dispositifs optiques de visée et ceux qui permettent à l'image de se former correctement sur le plan de la pellicule ou de la cible électronique, ces outils fonctionnent 
selon le principe fondamental de la mise en perspective que Paul Rossier ${ }^{3}$ définit ainsi : La perspective A' d'un point A de l'espace est l'intersection du plan du Tableau (T) sur lequel on veut représenter le point, avec le Rayon de visée (RV) issu du Point de visée (PV), joignant le point A dont on cherche la perspective.
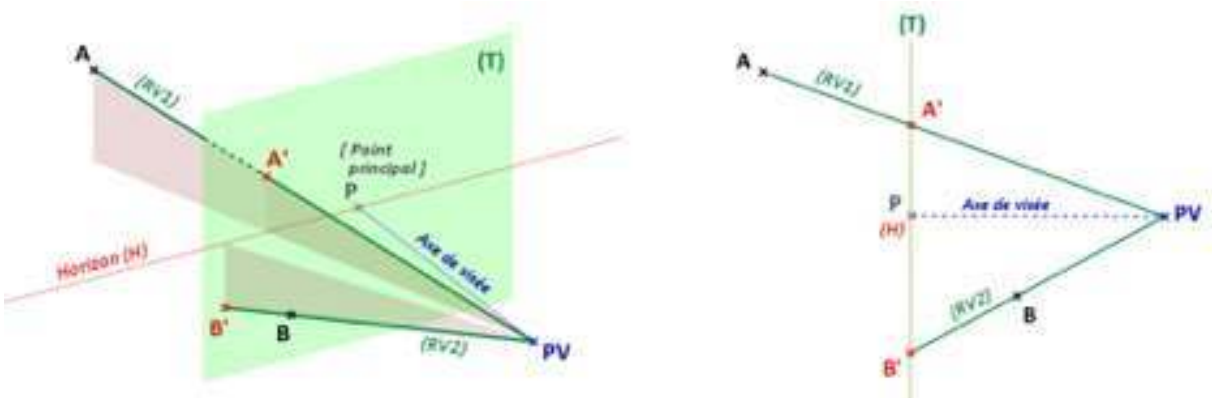

14 La transformation géométrique ainsi définie, opérant la contraction d'un espace géométrique à 3 dimensions (volume) sur un espace géométrique à 2 dimensions (le plan de l'image), met en perspective tous les volumes et espaces selon des principes d'organisation spatiale du plan image mis en évidence pour la première fois au début des années 1400 par un jeune orfèvre florentin, Filippo Brunelleschi. Ce dernier a réalisé deux expériences qui ont pour cadre des sites de la ville de Florence. Elles sont constitués de deux panneaux peints, aujourd'hui disparus, qui exemplifient la construction perspective d'une image peinte. Nous connaissons ces expériences par la description faite par son biographe, Antonio Manetti, plusieurs dizaines d'années plus tard.

Pour la première expérience, Filippo Brunelleschi avait peint sur une tavoletta (panneau de bois) d'une trentaine de centimètres de côté, le Baptistère qui fait face au portail de la cathédrale Sainte Marie des Fleurs. Le bâtiment était peint tel qu'on pourrait le voir si on se plaçait dans l'encadrement du portail de la cathédrale. Le panneau était percé d'un trou à travers lequel on regardait l'image peinte du baptistère réfléchie dans un miroir que l'on tenait à bout de bras.

Selon des historiens de l'art, lorsque l'on escamotait le miroir on pouvait vérifier que l'image peinte correspondait bien au bâtiment du Baptistère que l'on découvrait derrière le miroir. Cette interprétation de l'expérience sous-entend que le miroir n'aurait été introduit qu'après tracé du bâtiment sur le panneau et le perçage du panneau aux seules fins de vérification de la correspondance avérée du dessin et du bâtiment modèle. Compte tenu de la complexité du raisonnement et de la démarche nécessaires pour imaginer un tel dispositif (image peinte à l'envers sur la tavoletta et détermination précise de la position du trou notamment) alors que la perspective est à peine naissante, nous préférons une autre hypothèse : le tracé du baptistère sur le panneau peint pourrait être issu d'un dispositif expérimental dans lequel le miroir a servi à concevoir le tracé de la perspective. En effet, si on regarde à travers un panneau de bois percé un échiquier posé devant un miroir posé verticalement face à nous, on peut tracer sur le miroir l'échiquier en perspective.

17 L'image que l'on vient de tracer se superposant dans le miroir avec le reflet du panneau de bois, on constate également assez rapidement que le même effet pourrait être obtenu si on reportait ce dessin sur le panneau le panneau de bois4. 


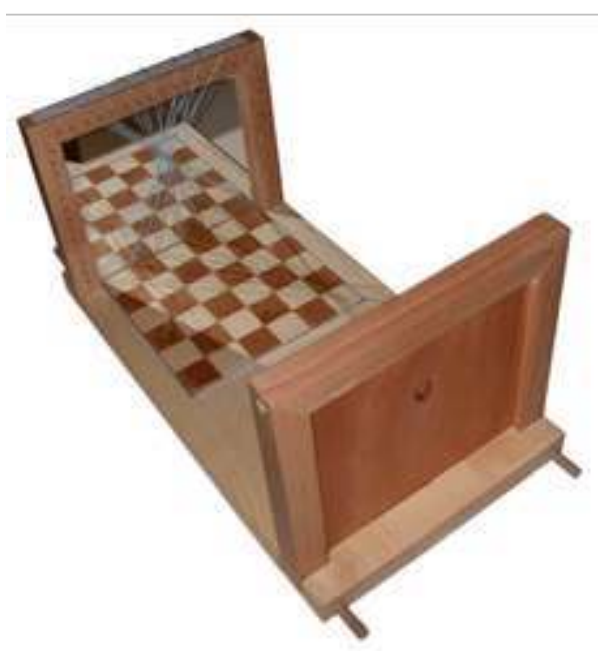

Ainsi, le miroir pourrait avoir été présent dès le début de l'expérience et avoir rendu possible le tracé du volume du bâtiment à partir d'un report de ses dimensions au sol et des différentes hauteurs sur les côtés. Cela résout aussi beaucoup plus simplement le problème de l'emplacement du trou dans le panneau de bois puisque c'est précisément à travers lui que l'on regarde et que l'on trace l'image pour comprendre et construire. En effet, lorsqu'on regarde le dessin qui reproduit le reflet de l'échiquier dans le miroir, on constate que les droites du plan qui sont perpendiculaires au plan du miroir convergent toutes vers un seul et même point, le reflet du trou à travers lequel on regarde dans le miroir.

On peut penser que c'est de cette manière qu'est apparu le point de fuite central qui va dominer la perspective occidentale pendant une très longue période.

Pour la seconde expérience, telle qu'elle nous est présentée par Antonio Manetti, Filippo Brunelleschi s'est affranchi du miroir. Pour installer cette nouvelle expérimentation visuelle, il a peint sur un panneau de bois le Palazzo Vecchio de la place de la Seigneurie et ses proches abords tels qu'ils seraient perçus depuis l'angle opposé de la place. Il a découpé le panneau peint suivant la silhouette des bâtiments dans le ciel et l'a posé au coin de la place à partir duquel sa représentation était supposée avoir été peinte. Si l'observateur se plaçait à l'endroit du point de visée qui a servi de centre à la transformation perspective, il pouvait constater que l'image peinte et sa silhouette coïncidaient avec le bâtiment et ses abords derrière le panneau.

21 Avec cette seconde expérience, Filippo Brunelleschi a très précisément installé, au début du Cinquecento, le dispositif expérimental qui sera décrit quelques siècles plus tard par la définition de la perspective citée ci-dessus : une fois le point de visée fixé et défini le tableau (plan sur lequel on va tracer la mise en perspective ${ }^{5}$ ), chaque point du tableau est le résultat de l'intersection du plan du tableau avec le rayon de visée issu du point de visée et joignant le point du bâtiment dont il est la perspective.

Ces deux expériences de Filippo Brunelleschi mettent en lumière plusieurs grands principes fondateurs de la construction perspective d'une image plane :

- La perspective ne se révèle à l'œil de l'observateur que s'il parvient à fixer son regard en contraignant la visée à l'aide d'un dispositif réduisant l'œil à un trou le plus étroit possible.

- L'axe de visée doit être fixe pour que le tracé soit le plus précis possible. 
- Le point de visée est fixe et unique pour que l'on travaille toujours sur la même image.

Dans ces conditions,

- Les droites perpendiculaires au tableau sont représentées par des droites convergeant toutes vers un seul et même point, le point principal (le reflet du trou sur le miroir).

- Les droites parallèles au plan du miroir (le tableau perspectif) sont représentées par des droites de même direction, notamment les droites horizontales (représentées par des horizontales dans le dessin) et les droites verticales (représentées par des verticales). Des droites parallèles dont l'écartement est constant dans l'espace sont représentées par des droites dont l'intervalle diminue au fur et à mesure que l'on se rapproche du point de fuite principal ; la droite la plus proche représentant la droite la plus éloignée du plan du tableau.

Le dispositif expérimental mis en œuvre par Filippo Brunelleschi est analogue à nombre d'autres procédés, méthodes ou outils décrits par ailleurs. Les "portillons » de Dürer présentent par exemple différentes méthodes pour fixer le point de visée et matérialiser le rayon de visée qui va traverser le plan du tableau, lieu du dessin. L'instrument de Tomaso Laureti rapporté par Egnatio Danti ${ }^{6}$ permet de vérifier à partir d'une règle verticale fixant le point de visée et à travers un cadre en bois qu'un rectangle divisé en quatre bandes égales sera représenté selon les principes de la perspective tels que mis en évidence précédemment. Philippe Comar montre un dessin de Léonard de Vinci, La paroi de verre (Milan, Bibliothèque Ambrosienne), où « l'œil maintenu par un viseur, Léonard de Vinci s'applique à représenter une sphère sur une paroi vitrée $»^{7}$.

La camera oscura a fait partie de ces multiples appareils qui permettaient de tracer des perspectives. Les planches IV et V du chapitre dessein de l'Encyclopédie de Diderot et d'Alembert en donnent plusieurs versions et usages. Des érudits arabes comme Ibn AlHaytham (965-1039) la connaissent et l'ont utilisée pour différentes expériences sur la lumière ${ }^{8}$. Elle a donc servi à dessiner selon les règles de la perspective mais, parallèlement, elle se transformera tout à la fois en outil de projection et en appareil de prise de vue. Pour cette dernière application, il faudra que Daniele Barbaro (Venise, 1514-1570) place une lentille devant le sténopé qui put s'agrandir puis qu'au début du XIXe siècle Nicéphore Niepce pose une plaque photosensible sur la face arrière de la boite noire recevant l'image projetée. L'image animée donnant naissance au cinéma sera ellemême le produit du défilement discontinu d'une succession d'images fixes.

\section{Les dispositifs de représentation audiovisuelle}

Quel que soit l'appareil de prise de vue que l'on utilise, on peut considérer que l'on met en œuvre un dispositif perspectif constitué d'un tableau (surface plane sur laquelle va se former l'image), d'un axe de visée qui lui est perpendiculaire à l'extrémité duquel se trouve le point de visée centre de la transformation perspective à partir duquel va s'organiser chaque image. Chaque fois que l'on bougera la position du point de visée ou la direction de l'axe de visée, on obtiendra une nouvelle image. Dans le cas d'un mouvement d'appareil, chaque image saisie sera sémantiquement liée aux autres mais avec sa construction et son autonomie propres. 

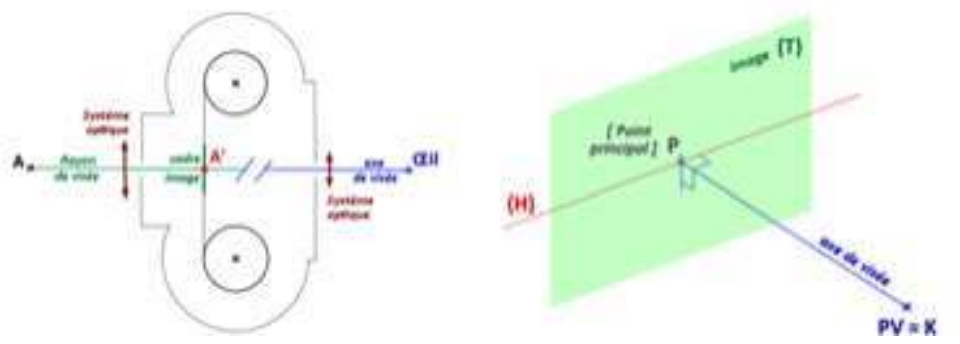

Les outils de représentation audiovisuelle fragmentent l'espace et le temps. Le dispositif perspectif que Leon Batista Alberti qualifiait de «fenêtre ouverte par laquelle on puisse regarder l'histoire $»^{9}$ est borné. Seul l'espace compris dans le cadre est saisi et, pour le cinéma, c'est l'assemblage des différents fragments visuels collectés associés à des sons particuliers qui va produire le sens voulu par le réalisateur. Il y a donc ce qui est vu et ce qui ne l'est pas. C'est généralement dans les parties non vues que l'on va pouvoir insérer les différents éléments du dispositif de tournage qui permettra de réaliser les images voulues, notamment pour le décor.

Le travail du décorateur de films, contrairement à l'architecte qui doit concevoir un espace continu dans lequel on va circuler, doit tenir compte de cette fragmentation visuelle spécifique aux représentations audiovisuelles de l'espace. Il va pouvoir s'appuyer sur cette spécificité pour imaginer et mettre en œuvre des dispositifs particuliers qui lui permettront de répondre au programme (ou cahier des charges) proposé par le réalisateur avec lequel il travaille.

Si l'on reprend la classification élaborée par Éric Rohmer ${ }^{10}$, la construction audiovisuelle mobilise trois catégories d'espaces distincts : le cadre de l'image qu'il appelle «espace pictural», le lieu de tournage qu'il nomme «espace architectural» et le produit du montage qu'il définit comme "espace filmique». C'est l'ensemble de ces catégories d'espace que doit maîtriser le réalisateur tout au long du processus de réalisation de son projet audiovisuel.
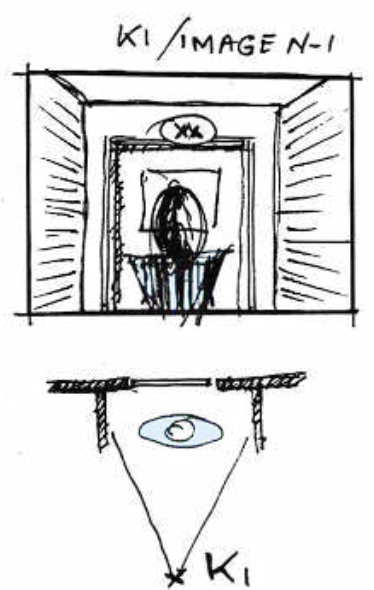
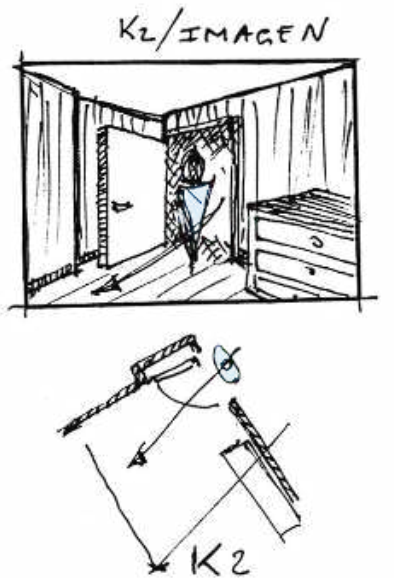
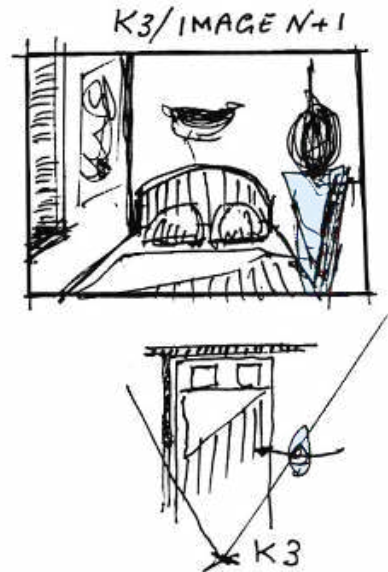

Lors de la phase de conception tout d'abord, le réalisateur va construire ses images et imaginer comment les assembler entre elles, comment les associer à des sons et des entrelacs sonores. Celles et ceux dont il a besoin pour produire le sens voulu par lui et qui lui sera propre. Chaque image, chaque photogramme de son film est le lieu de la perspective sur laquelle il va pouvoir prendre appui pour déterminer avec les techniciens qui vont faire le film avec lui la manière de fabriquer toutes ces images. C'est à ce 
moment qu'il décidera avec eux si les images seront tournées dans des décors ou dans des lieux déjà existants et à quel point elles seront composites en intégrant plus ou moins d'effets spéciaux. Il va donc élaborer une collection d'images éparses qui pourront être collectées dans des espaces de différentes natures, dans des lieux qui pourront être contigus ou non. La continuité spatiale n'est plus une contrainte. À la demande du réalisateur le décorateur va concevoir les différents décors nécessaires mais il va aussi pouvoir proposer différentes solutions de fragmentations visuelles et spatiales qui pourront être différentes à la fois de la continuité spatiale que nous vivons quotidiennement et de l'espace que produira le film.

C'est sur les différents lieux de tournage que l'on va fabriquer chacune des images nécessaires à la confection du film. C'est le lieu de la mise en œuvre des dispositifs techniques décidés antérieurement pour obtenir les images attendues. La caméra peut changer de place ou d'axe après chaque plan cinématographique en fonction des images conçues préalablement. Le décorateur va pouvoir prendre en compte cette discontinuité des plans pour organiser, si nécessaire, la discontinuité des décors et de l'espace de tournage. Il ne construira que ce dont le réalisateur a besoin pour le film. De même qu'il va pouvoir utiliser toutes les propriétés de la construction perspective des images qui vont être fabriquées. Si l'image est composite, c'est-à-dire le résultat de la combinaison de plusieurs dispositifs distincts, l'organisation perspective de l'image dans le plan va notamment permettre les associer correctement les uns aux autres. Ainsi, avec la perspective les différents composants coïncideront les uns avec les autres dans le plan de l'image et les intervalles entre eux disparaitront. Chaque technicien concerné par tout ou partie de l'image produite restant attentif à ce que tous les éléments dont il a la charge participent pleinement et parfaitement la construction d'une image unique et unifiée. Les parties faisant face à la caméra étant les seules à être vues, tous les éléments constructifs des dispositifs utilisés pourront être mis en place dans les parties non vues à l'arrière des éléments du décor et hors du cadre.

31 Après le tournage, le réalisateur va donc disposer d'une série de fragments visuels et sonores qu'il va devoir agencer pour donner forme à son film. C'est le résultat de son montage qui va donner la cohérence aux lieux qu'il a créés. À l'instar de Dziga Vertov ${ }^{11}$ qui créait « une chambre très extraordinaire » composée de « douze murs [...] pris dans les différentes parties du monde ", ce montage va donner naissance à une composition architectonique particulière. Ce nouvel espace résulte donc de l'assemblage des images et

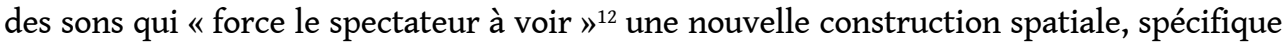
de chaque film. En conséquence, si le réalisateur maitrise l'ensemble du processus de réalisation audiovisuelle, de la conception au montage ${ }^{13}$, il est le véritable maittre d'œuvre des différentes compositions architectoniques de son film qui vont constituer l'espace spécifique construit par son film. C'est sa seule garantie pour que «son œuvre forme un tout cohérent $»^{14}$ dans un domaine artistique où il est presque toujours contraint de travailler avec une équipe de techniciens qui, même s'ils se sont mis à son service, ne peuvent avoir la vision globale qu'il a lui-même de son œuvre.

\section{Un outil d'aide à la conception destiné à pré-voir}

La perspective exposée au monde par Filippo Brunelleschi, sera formulée par écrit par Leon Battista Alberti quelques années plus tard et Piero della Francesca l'installera de façon méthodique et raisonnée avant la fin du Cinquecento. Pour Williams M. Ivins ${ }^{15}$ la 
perspective centrale et l'essor qu'elle a rapidement connu prendront une place importante dans la conquête du monde et son exploration parce qu'elle produit des images dont l'aspect est très proche de la perception visuelle et qu'elle est fondée sur une logique rigoureuse permettant d'obtenir des codes visuels constants rendant possible la sédimentation des savoirs $t$ des explorations donnant lieu à relevés et représentations dessinées ou peintes. Mais, ce nouveau mode de représentation du monde qui se perfectionnera tout au long des siècles qui ont suivi (géométrie projective avec Girard Desargues, géométrie descriptive avec Gaspard Monge, développement des modèles numériques aujourd'hui) garde la trace de la première expérimentation de Filippo Brunelleschi : une représentation du monde centrée sur l'œil (le reflet dans le miroir) qui est au fond de chaque image.

Si la perspective centrale s'est fortement chargée de sens par la suite ${ }^{16}$, Filippo Brunelleschi en a fait un usage propre. Il n'est pas peintre mais orfèvre. Au moment où il réalise ses expériences de perspective, il est en train de basculer vers l'architecture. Son intérêt pour la perspective n'est sans doute pas étranger à ce nouveau domaine qu'il explore. Artisan orfèvre, il façonne ses créations avec ses mains. Il les pense avec ses mains. L'architecture qu'il expérimente, les projets de bâtiments qu'il veut concevoir ont des caractéristiques propres qui contreviennent à cette manière de faire et le dépossèdent de toute la richesse de cette pensée manuelle ${ }^{17}$ qu'il pratique à la perfection. Il veut sans doute continuer à penser l'architecture avec ses mains et il recherche des outils qui lui feront retrouver sa dextérité d'orfèvre. Or, en passant de l'orfèvrerie à l'architecture, il est confronté à un problème d'échelle. Pour intéressant qu'il puisse être, le travail de la maquette suppose le plus souvent que le projet de bâtiment existe déjà. Pour concevoir un bâtiment, il a besoin de le voir de sa globalité.

Avec le dessin en perspective, il peut continuer à penser l'architecture avec ses mains. Elle est un moyen qui lui permet de pré-voir ses projets d'architecture, de valider ses hypothèses de travail en visualisant le projet dans son entier et, si nécessaire, d'y pénétrer avant même qu'il existe.

En mettant au point le dispositif expérimental de ses deux démonstrations de perspective, il forge l'outil d'aide à la conception dont il a besoin.

Dans cette logique, lorsqu'il réalise ses expériences, Filippo Brunelleschi ne cherche pas à reproduire le monde. Il forge le nouvel outil dont il a besoin pour s'assurer de la validité de sa méthode de travail. Il prend l'exemple de bâtiments existants pour vérifier que les images en perspective de bâtiments qu'il produit rendent bien compte des bâtiments tels qu'ils seront. La perspective est alors, pour cet architecte en construction, un outil d'anticipation et de simulation qui va l'aider dans son travail de conception des projets d'architecture.

Cette démarche opère dans le même temps une transformation notable des pratiques professionnelles au sein des métiers du bâtiment. Les corporations du bâtiment ont leurs propres règles et principes de fonctionnement. La conception et la construction des bâtiments obéissent elles-mêmes à des modes de production codifiés et principalement fondées sur l'expérience des bâtisseurs qu'ils reproduisent et perfectionnent au fil des constructions. Avec ce nouvel outil d'aide à la conception, Filippo Brunelleschi extraie la conception du chantier et instaure par la même une phase d'élaboration préalable au chantier. C'est au cours de cette dernière qu'il va pouvoir concevoir les espaces, appréhender leur qualité, penser les modalités de mise en œuvre des matériaux, imaginer 
les systèmes constructifs pour créer des projets architecturaux novateurs comme la coupole de la cathédrale de Florence dont le tambour octogonal au-dessus de l'autel est d'une telle dimension que personne ne savait vraiment comment le couvrir. En travaillant sur l'élaboration de ce nouvel outil de travail du concepteur et en installant le travail de l'architecte bien en amont de la construction, Filippo Brunelleschi se donne les moyens de maîtriser l'œuvre. Il ouvre dans le même temps la voie à l'émergence du métier d'architecte maitre d'œuvre tel que nous le connaissons aujourd'hui, c'est-à-dire celui qui conçoit, dans un premier temps, un projet de bâtiment au cours d'une phase indispensable d'élaboration préalable et qui assure ensuite la conduite du chantier pour s'assurer que le bâtiment réalisé sera en tous points conforme à ce qu'il a imaginé et prescrit.

\section{Un bâtiment n'est pas un film}

La démarche du réalisateur de films que nous proposons et défendons à l'ESAV est fondée sur la maitrise de l'ensemble du processus de réalisation audiovisuel. Le réalisateur est le maître d'œuvre qui conçoit le film, imagine les agencements d'images et de sons, puis qui dirige le tournage et les opérations de montage de son film dont il est le seul à savoir intimement comment il doit être dans sa globalité.

L'architecte et le réalisateur sont donc tous deux maitres d'œuvre. Il conçoivent leurs projets et définissent les conditions de leur réalisation. Selon des modalités différentes, il constituent les équipes de professionnels et techniciens qui vont réaliser leurs projets. Le plus généralement, ils dirigent la fabrication de leurs œuvres pour s'assurer qu'elles soient réalisées selon leurs prescriptions. Et, une fois l'œuvre réalisée, elle leur échappe pour être totalement appropriée par les utilisateurs ou les habitants (architecte) et les spectateurs (réalisateur).

On peut donc trouver une réelle analogie entre le travail du réalisateur et celui de l'architecte. Lorsqu'il créé sa chambre extraordinaire au début de siècle dernier, Dziga VERTOV proclame : «je suis un constructeur ${ }^{18}$. Dans le courant des années soixante, en construisant une ville pour son film Playtime, Jacques Tati a tenté d'approcher la stature de l'architecte et il en avait revendiqué le statut avec le générique du film Mon Oncle ${ }^{19}$. Au lendemain de la seconde guerre mondiale, Philippe Fauré-Frémiet ${ }^{20}$ prenait appui sur le métier d'architecte qu'il qualifie de "maître constructeur, celui qui dresse le plan et dirige la construction", pour défendre l'idée que le cinéma devait s'inspirer de cette profession pour se reconstruire et rompre avec la conception marchande qui prédomine dans l'industrie cinématographique. Il réclamait qu'à l'instar de l'architecte le réalisateur soit celui qui « surveille tout, dirige, exige, corrige " pour que «l'œuvre terminée [soit] sienne » et qu'elle soit "la réalisation exacte, totale de sa pensée ». Aujourd'hui, on constate un mouvement inverse. Sans doute attirés par la notoriété des gens de cinéma, leurs gains (pour certains) et leur capacité à mobiliser les médias, les architectes regardent fréquemment du côté du cinéma. Ils cherchent à s'approprier de nouveaux outils de représentation pour prendre la mesure du monde et recherchent dans les modes de construction cinématographiques de nouvelles sources d'inspiration pour créer leurs bâtiments.

41 Mais l'analogie ne peut aller trop loin car elle provoquerait des contresens majeurs. Même si les mots sont les mêmes, séquence, plan, montage par exemple, ils ne désignent pas du tout les mêmes concepts ni les mêmes processus. Certes l'architecture et le cinéma 
sont des arts complexes pour lesquels on peut trouver nombre de similitudes mais les objets qui sont réalisés pour l'une et pour l'autre différent grandement. Si les méthodes et processus de création peuvent se comparer et se nourrir l'une l'autre, dès qu'une analogie apparait il est important de s'interroger sur les différences et comprendre ce qui différencie le travail et les productions des uns et des autres. Étudier ces différences peut donner à chacun des indications précieuses sur ses propres pratiques de création.
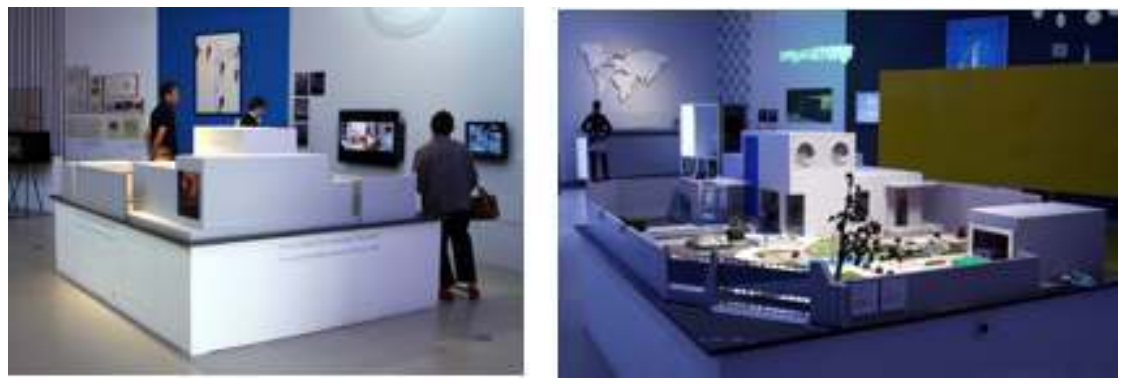

Exposition « Jacques TATI, deux temps, trois mouvements... » Cinémathèque française, 2009 • Photos Jean-Luc Antonucci

Côté caméra et face cachée (ou non vue) : la Villa Arpel de « Mon Oncle » n'existe que pour le film.

\section{Une fin qui échappe}

Les questions relatives à l'espace, à son organisation et à sa gestion, rapprochent les architectes et les réalisateurs. La perspective qui est un outil d'aide à la conception pour les uns est une caractéristique intrinsèque des images qu'ils produisent pour les autres. Dans le processus de réalisation audiovisuelle, le décorateur est à l'intersection de l'architecture et des représentations audiovisuelles. Il intervient à la demande d'un réalisateur pour définir des dispositifs décor qu'il devra ensuite mettre en œuvre pratiquement sur un plateau de tournage. Mais, tout son travail est tourné vers l'objectif de la fabrication des images qui constitueront la matière première du film qui reste celui du réalisateur. Le décor deviendra ce que ce dernier en fera.

Ceux qui travaillent sur l'espace sont toujours à la recherche d'outils susceptibles de les aider à créer. Certains ont entrepris de forger les outils qui leur manquaient. Sans que l'on puisse en avoir encore la certitude, avec l'exploration de la perspective Filippo Brunelleschi est très probablement l'un d'eux. Bien d'autres après lui ont contribué à la fabrication d'outils permettant de mieux comprendre l'espace, de mieux l'explorer et le représenter. Girard Desargues (1591-1661) qui était également architecte a ouvert par exemple la voie à la géométrie projective. En 1869, l'architecte Henry Revoil a déposé un brevet pour le téléiconographe, instrument de relevé permettant de dessiner des objets à distance éloignée. L'architecte Eugène Viollet-Leduc l'a utilisé pour réaliser la cartographie du Mont Blanc qu'il se proposait de restaurer. Ce dernier n'a pas cessé tout au long de sa carrière de mettre à profit toutes les ressources possibles à son époque pour avoir les moyens de s'approprier le savoir-faire et les intentions des bâtisseurs qui l'avaient précédé, notamment ceux du Moyen-Âge ${ }^{21}$. Il utilisera par exemple la planchette photographique d'Auguste Chevallier, chambre photographique produisant des panoramiques anamorphosés de forme circulaire, pour le château de Pierrefonds, mais aussi, en d'autres occasions, la chambre claire de William Hyde Wollaston.

Lorsqu'au début de sa carrière, l'architecte Robert Mallet-Stevens participe à la réalisation de films ${ }^{22}$, il conçoit avec le décorateur de cinéma Jean Perrier, un tracé de 
perspective qui permet d'obtenir une image en fonction de la position de la caméra, des objectifs dont elle sera équipée et du format de la pellicule utilisée. Ce dispositif, enseigné par le décorateur Hugues Laurent à l'IDHEC ${ }^{23}$, est constitué du plan et des façades du décor organisés autour du cadre image; les différentes projections du tableau étant confondues avec les côtés du cadre image sur lequel ils s'appuient. Les tracés s'effectuent à partir des projections du point de visée placé sur l'axe de visée et confondu avec la position et la hauteur de la caméra sur le plan et les élévations. Dans chacune des projection (plan et élévations), sa distance par rapport au plan du tableau correspond à la distance focale de l'optique dont sera équipée la caméra, rapportée aux dimensions du cadre image. La perspective est tracée directement dans le cadre image à partir des tracés dans les différentes projections disponibles (plan et élévations). Ce dispositif a notamment pour avantage de voir très rapidement en fonction de la position de la caméra et des optique choisies, les parties qui seront dans le cadre et celles qui ne seront pas vues. C'est un dispositif qui met en œuvre pratiquement la fragmentation visuelle et spatiale. Il donne au décorateur et au réalisateur la possibilité d'explorer, en amont de la construction d'un décor, ses ressources potentielles et l'utilisation qui peut en être faite.

Aujourd'hui, les outils numériques qui sont à notre disposition ouvrent de nouvelles perspectives. Sans remettre en cause l'essence de chacun des métiers concernés, ils modifient les pratiques professionnelles, les modalités de conduite d'un projet et les conditions de coopération entre les différents intervenants qui participeront à sa réalisation. Cela doit inciter architectes, décorateurs et réalisateurs à investir ce domaine, pour comprendre ces transformations mais aussi pour les maitriser.

Pour les architectes, la maquette numérique et les outils de management numérique de projets $^{24}$ constituent un réel enjeu pour le devenir de la profession. Ces outils qui imposent une technicité nouvelle et accrue, permettent par superposition d'objets numériques relevant des divers intervenants de l'acte de construire, de donner une image complète, c'est-à-dire constructive, du bâtiment tel qu'il sera réellement mis en œuvre. De plus, les objets numériques pouvant être transmis et enrichis dans le temps, ces technologies ouvrent sur la gestion de patrimoine, l'entretien et la maintenance des bâtiments par exemple. La maitrise de ces outils déterminera à terme si les architectes restent ou non les véritables maîtres d'œuvres de leurs projets.

Pour les professionnels du cinéma, l'immixtion du numérique à tous les niveaux de la production des films transforme aussi les pratiques et les métiers en profondeur. Le travail de décorateur ne peut s'envisager aujourd'hui sans une connaissance approfondie des outils numériques pour travailler le décor (conception, élaboration avec le réalisateur, implantation des décors, documents de construction, etc.) mais aussi pour intégrer la production des décors numériques qui prennent une place de plus en plus importante dans la production cinématographique (avec des interactions qui peuvent être fortes pendant le tournage et pendant la phase de post-production). À la vue de certains films, on s'étonne de constater quel niveau de maîtrise on a désormais atteint pour la production numérique de décors qui ne seraient plus à portée de ce que l'on peut mettre en œuvre sur un plateau. Parallèlement et en raison de cette maitrise, la capacité de détruire bâtiments, villes, vaisseaux et véhicules de toutes natures, n'a jamais été aussi forte. Certains blockbusters en usent et abusent jusqu'à l'indigestion! Tous les secteurs de la production cinématographique sont concernés. Techniques et outils se diversifient tout en convergeant progressivement les uns vers les autres. Cela condamne le réalisateur qui veut conserver la maîtrise de son œuvre d'être « comme l'architecte, à quelque rang que 
son talent personnel le classe, un homme [ ou une femme ] pleinement instruit de toutes les ressources du "métier" » ainsi que l'écrivait Philippe Fauré-Frémiet ${ }^{25}$.

L'imagerie numérique, le développement des interconnexions de toutes natures entre les différents systèmes et domaines d'intervention engagent les réalisateurs et les architectes à se donner les moyens d'une appropriation rapide des outils numériques et de leurs divers usages. C'est une garantie pour qu'ils restent maitres de leurs œuvres. C'est également à la faveur de ce processus d'appropriation que leurs besoins peuvent susciter la création d'applications nouvelles pour lesquelles certains d'entre eux pourront créer, ou participer à la création, des nouveaux outils qui leur seront nécessaires pour aller plus avant dans l'amélioration du processus de création.

\section{NOTES}

1. PRÉDAL René, Hugues Laurent, un demi-siècle d'évolution du décor de cinéma, dans l'AVANT SCÈNE CINÉMA n 192, septembre 1977.

2. . D'autres décorateurs ont travaillé sur ce lien entre décor et perspective. Par exemple Robert et Nonce GIORDANI, La perspective dans l'image, Éditions Dujarric, Paris, 1987.

3. ROSSIER Paul, Perspective, Éditions du Griffon, Neufchatel, 1946 - 3ème édition 1965

4. . Pour un développement détaillé de cette question, on peut se reporter à un article du numéro 5 de la Revue Entrelacs du LARA, La Machine : Jean-Luc ANTONUCCI, Perspective en construction, p. 38-52 [ http://entrelacs.revues.org/162 ]

5. . Pour Leon Battista ALBERTI un voile intersecteur concrétise ce plan. Leon Battista ALBERTI, De Pictura (1435) / De la Peinture, traduction de Jean-Louis SCHEFER, Macula, Dédale, Paris, 1992, p. 147

6. DANTI Egnatio, Le due regole della prospettiva pratica da Vignola, avec les commentaires d'E. Danti, Rome, 1583

7. COMAR Philippe, La perspective en jeu. Les dessous de l'image, Découvertes Galimard, $\mathrm{n}^{\circ} 138$, Paris, 1992

8. 8 Voir notamment Gérard SIMON, Archéologie de la vision, Seuil, Paris, 2003

9. ALBERTI Léon Baptista, Ibidem, p. 115

10. ROHMER, L'organisation de l'espace dans le Faust de Murnau, Ramsay Poche Cinéma, Union Générale d'Éditions, Paris, 1977, p. 11.

11. VERTOV Dziga, Articles, journaux, projets, 10/18, Union Générale d'Éditions, Paris, 1972, pp. 28-30

12. VERTOV Dziga, Ibidem

13. Principe que défend l'ESAV

14. ROHMER Éric, Ibidem, p. 12

15. IVINS William M., La rationalisation du regard, préface du livre On the rationalism of sight republié en 1973 par Plenum Press, New York. Traduction publiée dans la revue Culture et Technique, $\mathrm{n}^{\circ}$ 14, 1985, pp. 31-37.

16. Voir notamment Erwin PANOFSKY, La perspective comme forme symbolique, Les éditions de Minuit, Paris, 1975

17. LEROI-GOURHAN André, Les racines du monde, Entretiens avec Claude-Henri Rocquet, Belfond, Paris, 1982, p. 67 
18. VERTOV Dziga, Ibidem, p. 29

19. ANTONUCCI Jean-Luc, Jacques TATI, constructeur, publié par le site Cadrage.Net [ http:// www.generique-cinema.net/analyses/tati.html ]

20. FAURÉ-FRÉMIET Philippe, $L^{\prime}$ « architecte » de cinéma dans la Revue du Cinéma, n 15, juillet 1948, Paris, pp. 44-51

21. Voir notamment le catalogue de l'exposition présentée récemment par la Cité de l'architecture \& du patrimoine : Laurence de FINANCE et Jean-Michel LENIAUD (sous la direction de), VIOLLET-LE-DUC, Les visions d'un architecte, Cité de l'architecture \& du patrimoine, Éditions Norma, Paris, 2014

22. Notamment L'Inhumaine de Marcel L'Herbier en 1924

23. IDHEC : Institut des hautes études cinématographiques créé en 1943 qui, en 1988, s'est fondue dans la Fondation européenne des métiers de l'image et du son (FEMIS).

24. Appelés aujourd'hui BIM (Building information management)

25. FAURÉ-FRÉMIET Philippe, Ibidem

\section{AUTEUR}

\section{JEAN-LUC ANTONUCCI}

Architecte, maître de conférences à l'ESAV, LARA - SEPPIA 